EPJ Web of Conferences 60, 09002 (2013)

DOI: $10.1051 /$ epjconf $/ 20136009002$

(C) Owned by the authors, published by EDP Sciences, 2013

\title{
Top mass and properties at the LHC and Tevatron
}

\author{
M.J.Costa on behalf of the ATLAS, CMS, CDF and D0 Collaborations ${ }^{1, a}$ \\ ${ }^{1}$ Instituto de Fisica Corpuscular (Centro Mixto Universidad de Valencia - Consejo Superior de Investigaciones Cientificas, Edificio \\ Institutos de Investigacion), C/Catedratico Jose Beltran 2. E-46980 Paterna, Spain
}

\begin{abstract}
An overview of the properties of the top quark measured with the ATLAS and CMS detectors at the LHC and with the CDF and D0 detectors at the Tevatron is presented. The latest and most precise measurements are shown, giving special emphasis to the top quark mass determinations. Measurements of the $b$-jet content in the top quark decay products and the $W$ polarisation, as well as a search for flavour changing neutral current processes in top quark decays are also reviewed.
\end{abstract}

\section{Introduction}

The top quark is the heaviest known fundamental particle, leading to important theoretical and experimental implications. The large top quark mass implies a large coupling to the Higgs boson and raises the possibility that it plays a special role in the mechanism of electroweak symmetry breaking. It also implies that the top quark decays rapidly through electroweak interactions before it can form a hadronic bound state, which provides the unique opportunity to study the properties from distributions of its decay products. It is therefore essential to measure with high precision top quark properties and confront them with the Standard Model (SM) predictions.

\section{Top quark mass}

Among all measurements performed at LHC and Tevatron, the top quark mass deserves special attention. It is a free parameter of the SM which together with the $W$ boson mass constrains the Higgs boson mass through electroweak quantum corrections, allowing for stringent tests of the SM.

The most precise determinations of the top quark mass performed so far have used one of the following methods:

- Template method: For a chosen observable sensitive to the top quark mass parameter $\left(m_{\mathrm{top}}\right)$, simulated distributions (templates) are produced using a number of discrete values of that parameter. These templates are fitted to distributions that interpolate between different input values of the top quark mass parameter. A fit to the observed data distribution is then performed to obtain the value of $m_{\text {top }}$ that best describes data.

- Ideogram method: The procedure defines a probability for observing the reconstructed top quark mass in

\footnotetext{
a e-mail: Maria.Jose.Costa@ific.uv.es
}

an event that is based on the mass resolution and an assumed input value of $m_{\text {top }}$. The probability for the signal is obtained through a convolution of a Gaussian for the mass resolution with a Breit-Wigner for the top quark decay, while the background probability is taken from the Monte Carlo (MC).

- Matrix Element: The full kinematic information of each event is used, by calculating per event probabilities for signal and background processes. The probabilities are calculated by integrating over the leading-order (LO) matrix element for $\bar{t}$ production folded with the parton distribution functions (PDFs) and transfer functions to describe the transition of parton momenta into measured momenta of the final state particles from top quark decays. The measured $m_{\text {top }}$ is then obtained by maximising the likelihood of the product of per event probabilities.

In all these methods the top quark mass determined from data corresponds to the mass definition as implemented in the MC. A dominant uncertainty comes from the jet energy scale uncertainty. For the case in which there is at least one $W$ boson decaying hadronically, the invariant mass of the two jets coming from the $W$ can be constrained to the known $W$ boson mass to reduce the impact of the jet energy scale on $m_{\text {top }}$. This comes with the cost of an additional increase of the statistical uncertainty which will be referred as JSF below.

The Tevatron experiments have recently provided a combination of 12 measurements performed by D0 and CDF collaborations in different channels including up to $8.7 \mathrm{fb}^{-1}$ of data [1]. This leads to a value of $m_{\text {top }}=$ $173.20 \pm 0.51$ (stat) \pm 0.36 (JSF) \pm 0.61 (syst) which is the most precise measurement to date and where the dominant uncertainties come from the jet energy scale and signal modelling.

The most precise inputs from CDF and D0 experiments correspond to measurements performed in the lepton+jets final state. The CDF measurement [2] uses the 
full available statistics of $8.7 \mathrm{fb}^{-1}$ and a template method with three observables: the reconstructed top quark mass for the best and second best jet to parton assignment and the reconstructed $W$ boson mass to constrain the jet energy scale. The result obtained is $m_{\text {top }}=172.85 \pm 0.52$ (stat) \pm 0.49 (JSF) \pm 0.85 (syst) GeV. The D0 measurement [3] uses instead a matrix element method and combines the results obtained for two independent data sets, leading to $m_{\text {top }}=174.94 \pm 0.83$ (stat) $\pm 0.53(\mathrm{JSF}) \pm 1.12$ (syst) $\mathrm{GeV}$.

At the LHC, the ATLAS [4] and CMS [5] experiments have also measured the top quark mass in various channels: lepton+jets, dilepton and all-hadronic final states using the full statistics of proton-proton collisions at $\sqrt{s}=7$ $\mathrm{TeV}$. The most precise measurements are also obtained in the lepton+jets channel.

The CMS measurement [6] employs an ideogram technique, using a kinematic fit of the decay products to the $t \bar{t}$ hypothesis and 2-dimensional likelihood functions for each event to estimate simultaneously $m_{\text {top }}$ and the jet energy scale factor. That leads to $m_{\text {top }}=173.49 \pm 0.27$ (stat) \pm 0.33 (JSF) \pm 0.98 (syst) $\mathrm{GeV}$, with the systematic uncertainty being dominated by the $b$ jet energy scale and colour reconnection uncertainties.

The ATLAS experiment has recently provided an updated measurement in the lepton+jets channel [7], leading to a very significant improvement (of around $40 \%$ ) with respect to the previous measurement [8]. The main sources of systematic uncertainties coming from the $b$ jet energy scale and the amount of initial and final state radiation (ISR/FSR) in $t \bar{t}$ events were better understood in Ref.[9] and Ref.[10] respectively. However, the largest improvement comes from the use of a 3-dimensional instead of a 2-dimensional template fit. In addition to the reconstructed top quark and $W$ boson masses, a new observable, sensitive to the $b$ to light jet energy scale, was introduced in the fit to simultaneously obtain $m_{\text {top }}$, a jet scale factor and a $b$ to light jet scale factor (bJSF). The result obtained for the top quark mass is then $m_{\text {top }}=172.31 \pm 0.23$ (stat) \pm 0.27 (JSF) \pm 0.67 (bJSF) \pm 1.35 (syst) GeV, where the dominant uncertainty now comes from the $b$-tagging efficiency.

Figure 1 shows the result of the ATLAS 3-dimensional analysis, together with that of the previous ATLAS 2dimensional measurement, the most precise measurements obtained by the CDF, D0 and CMS Collaborations and the result of the latest Tevatron combination described above. A combination of the top quark measurements provided by the different collaborations is in progress, for which a harmonisation in the treatment of systematic uncertainties and a proper understanding of the correlations is essential.

To attempt addressing the questions of whether there is any kinematical bias on the measurement of the top quark mass and whether the generators describe correctly the top quark mass observables in various regions of the phase space, the CMS experiment has measured the dependence of the top quark mass as a function of observables sensitive to different kind of important systematic uncertainties (namely colour reconnection, initial and final state radiation (ISR/FSR) and $b$ quark kinematics) [11]. Within the current precision no mismodelling is observed.

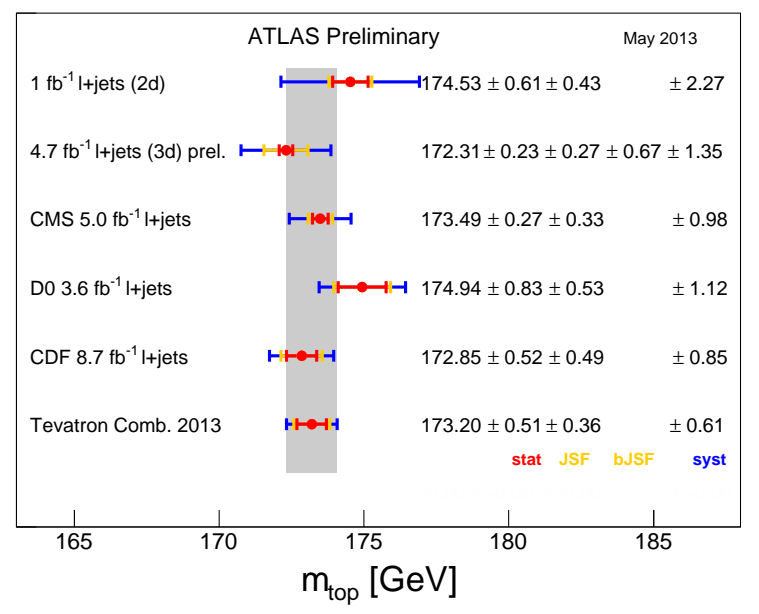

Figure 1. Summary of the most precise top quark mass measurements provided by CDF, D0, CMS and ATLAS collaborations [7], together with the previous ATLAS measurement and the Tevatron combination.

The measurement of the difference between the top/anti-top quark masses has also been performed at both Tevatron and LHC. The observation of a deviation from the null result would be a signature of CPT violation in the top quark sector. The latest and most precise measurement has been performed by the CMS experiment in the lepton+jets channel using the full statistics of protonproton collisions at $\sqrt{s}=8 \mathrm{TeV}$, leading to $\Delta m_{\text {top }}=$ $-272 \pm 196$ (stat) \pm 122 (syst) MeV [12] compatible with the SM.

\section{Top quark decay properties}

According to the SM the top quark decays nearly $100 \%$ of the time to a real $W$ boson and a $b$-quark. The experiments have measured the ratio of top quark branching fractions $R=B(t \rightarrow W b) / B(t \rightarrow W q)$. Figure 2 shows all measurements performed at Tevatron and LHC, all in agreement with the SM prediction, even if some tension was observed in the dilepton D0 measurement.

The latest and most precise measurement comes from the CMS experiment in the dilepton channel using data collected at $\sqrt{s}=8 \mathrm{TeV}$ [13]. The $b$ content is extracted from the number of $b$-tagged jets per event using a parametric model where all parameters of the model are measured from data. The result is in good agreement with the SM prediction: $R=1.023_{-0.034}^{+0.036}$ (stat + syst) and a lower endpoint for the confidence interval at $95 \% \mathrm{CL}$ is set using the Feldman-Cousins approach: $R>0.945$. The measurement is dominated by uncertainties on $b$-tagging, signal modelling and on the evaluation of the fraction of jets in the selected events which come from top quark decays.

The top quark decay $t \rightarrow Z q$, where $q=u$ or $c$, is highly suppressed in the SM by the GIM mechanism and occurs via quantum loop corrections, being of $O\left(10^{-14}\right)$. However, there are several models that predict enhancements where the branching fraction could be as large as 


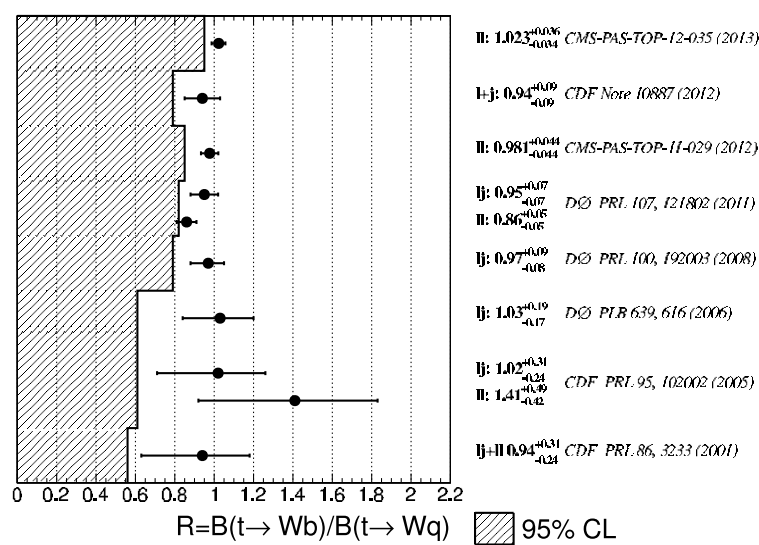

Figure 2. Chronological summary of the different measurements of R performed at the Tevatron and the LHC [13]. The shaded region represents the lower endpoint for the confidence interval at 95\% confidence level on R obtained after imposing the physical constraint $\mathrm{R} \leq 1$. The $\mathrm{CDF}$ measurement has now been published in Ref. [14]

$O\left(10^{-4}\right)$. The most stringent limit comes from the CMS measurement using data collected at $\sqrt{s}=8 \mathrm{TeV}$, setting an upper limit of $B(t \rightarrow q Z)<0.07 \%$ [15], which improves the previous ATLAS and CMS limits obtained using $7 \mathrm{TeV}$ center-of-mass energy collisions $[16,17]$.

\section{Probing the $W t b$ vertex}

Among the different top quark couplings to the gauge and Higgs bosons, the Wtb vertex deserves special attention, given that the top quark decays almost exclusively to a $W$ boson and a $b$ quark. These couplings can be probed by measuring the angular distribution of the lepton produced in a semileptonic top quark decay.

For the case of unpolarised top quarks, as produced in $t \bar{t}$ events, the only meaningful direction in the top quark rest frame is the $W$ momentum $(q)$. However, if the top quark is polarised (as occurs in the case of top quarks produced via the single top $t$-channel process) two additional directions can be considered: the transverse $T$ and the normal $N$ as defined in Ref. [18]. Therefore, three different angles can be defined between the charged lepton momentum in the $W$ rest frame and each of the three mentioned directions $q, T$ and $N$, as $\theta^{*}, \theta^{T}$ and $\theta^{N}$ respectively. The corresponding normalised differential decay rates can be written as:

$$
\begin{array}{r}
\frac{1}{\Gamma} \frac{d \Gamma}{d \cos \theta^{X}}=\frac{3}{8}\left(1-\cos \theta^{X}\right)^{2} F_{+}^{X}+\frac{3}{4} \sin ^{2} \theta^{X} F_{0}^{X}+ \\
\frac{3}{8}\left(1+\cos \theta^{X}\right)^{2} F_{-}^{N}
\end{array}
$$

where $X=(*, T, N)$ and $F_{i}^{X}$ are the $W$ polarisation fractions in the different mentioned directions (for the case of $X=*$ the so-called $W$ helicity fractions $F_{R}, F_{0}$ and $F_{L}$ ). Experimentally, one can either extract directly the $W$ polarisation fractions (e.g. using a template method) or unfold the

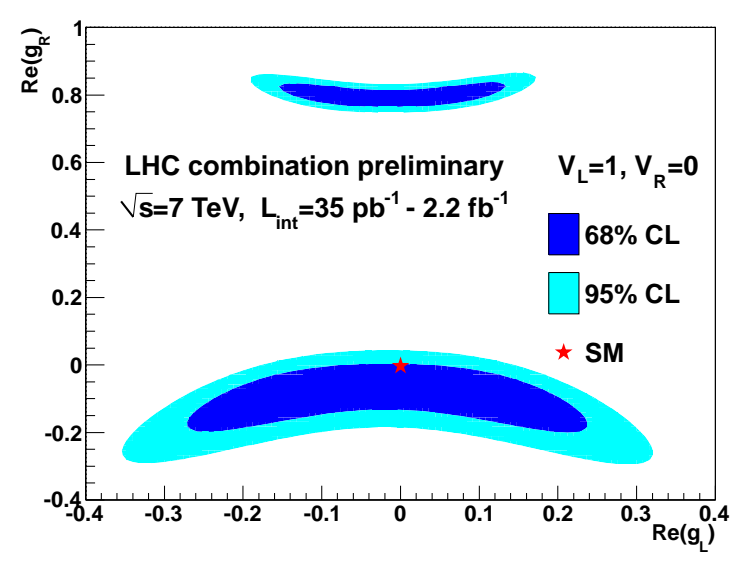

Figure 3. Allowed regions for the $W t b$ anomalous couplings using the combined helicity fractions and assuming $V_{L}=1$ and $V_{R}=0$, at $68 \%$ and $95 \%$ CL [19].

reconstructed angular differential rates to parton level to measure asymmetries.

While the $W$ helicity fractions and transverse polarisation (or the corresponding asymmetries) are only sensitive to the real part of $W t b$ anomalous couplings, the normal polarisation is sensitive to complex phases of the anomalous couplings.

The $W$ helicity fractions in $t \bar{t}$ events have been measured at both Tevatron and LHC, all measurements being in good agreement with the next-to-next-to-leading order (NNLO) pQCD prediction. The most precise determination comes from the first combination of the ATLAS and CMS results [19], based on measurements performed with data sets corresponding to integrated luminosities ranging from $35 \mathrm{pb}^{-1}$ to $2.2 \mathrm{fb}^{-1}$ produced at $\sqrt{s}=7 \mathrm{TeV}$ [20-22].

The combination is performed using the Best Linear Unbiased Estimator (BLUE) method [23, 24]. By construction, the sum of the three helicities is constrained to unity so that only two observables are independent. In this case measurements of $F_{0}$ and $F_{L}$ were combined, from which $F_{R}$ was extracted. The systematic uncertainties from the individual measurements are grouped into categories that are assumed to be independent. Defining the proper categories comprising sources of uncertainties that have similar origins and understanding the correlations between experiments and channels is crucial for such combinations. The results obtained are $F_{0}=0.626 \pm$ 0.034 (stat) \pm 0.048 (syst), $F_{L}=0.359 \pm 0.021$ (stat) \pm 0.028 (syst) and $F_{R}=0.015 \pm 0.034$ (stat + syst). Figure 3 shows the corresponding limits on the anomalous couplings $g_{L}$ and $g_{R}$, derived assuming the SM prediction for $V_{L}$ and $V_{R}$ and that the imaginary parts of all couplings are zero.

The above combination does not include a new measurement from CMS in the dilepton channel using the full available statistics at $\sqrt{s}=7 \mathrm{TeV}$ [25], nor a measurement performed in a single top topology using collisions at both $7 \mathrm{TeV}$ and $8 \mathrm{TeV}$ center-of-mass energies [26], both being compatible with previous results. 


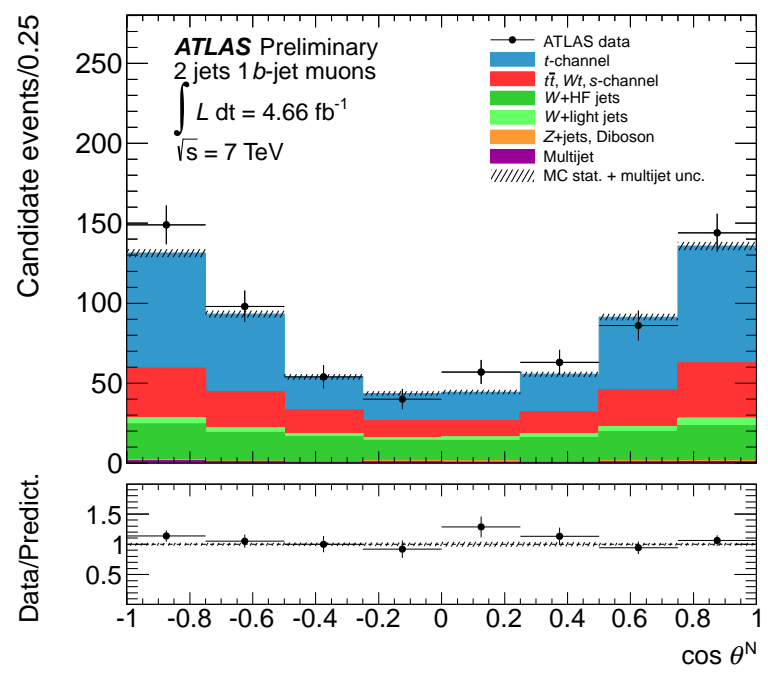

Figure 4. Reconstructed $\cos \theta^{N}$ angular distribution for the muon channel [28]. ATLAS data, simulated signal and different background contributions are shown. The uncertainties shown on the prediction take into account MC statistics and the normalization of the multijet background uncertainty.

The latest combination from the Tevatron experiments [27] based on data samples corresponding to integrated luminosities from 2.7 to $5.4 \mathrm{fb}^{-1}$ leads to $F_{0}=0.722 \pm$ 0.062 (stat) \pm 0.052 (syst) and $F_{R}=-0.033 \pm 0.034$ (stat) \pm 0.031 (syst).

Concerning the lepton angular distributions defined in the normal direction, it is in particular interesting to measure the forward-background asymmetry $A_{F B}$, as proposed in Ref. [18], because of its sensitivity to $\operatorname{Im}\left(g_{R}\right)$. A nonzero value of this asymmetry would automatically lead to a sign of $C P$ violation in top quark decays.

The ATLAS Collaboration has provided a first measurement of this asymmetry using single top quark $t$ channel produced events. Figure 4 shows the reconstructed $\cos \theta^{N}$ distribution for data together with the signal and background SM predictions. After background subtraction and unfolding data to parton level, the measured asymmetry is $A_{F B}^{N}=0.031 \pm 0.065$ (stat) ${ }_{-0.031}^{+0.029}$ (syst) [28]. Taking this measurement together with the theoretical prediction of the top quark polarisation, the first experimental limits on $\operatorname{Im}\left(g_{R}\right)$ are determined to be $[-0.20$, 0.30 ] at $95 \%$ confidence level. Both the measurement and the limit are in good agreement with the Standard Model.

\section{Summary}

The Tevatron and LHC experiments have entered the precision era for top quark properties. Tevatron analyses are now exploiting the full amount of data collected. At the LHC experiments, measurements have been provided with data collected at both 7 and $8 \mathrm{TeV}$ center-of-mass energies, even though the latter is not yet fully exploited for most measurements. So far all results are in good agreement with the SM predictions. Most measurements are however dominated by systematic uncertainties, mainly from signal modelling, jet energy scale and $b$-tagging uncertainties. The experimental challenge is then to keep improving in the understanding of the detector performance and perform measurements that allow theoretical models to be constrained, allowing for more stringent tests of the SM to be made and possibly observe deviations due to new physics.

\section{References}

[1] Tevatron Electroweak Working Group, CDF and D0 Collaborations, arXiv:1305.3929.

[2] T. Aaltonen et al. (CDF Collaboration), Phys. Rev. Lett. 109 (2012) 152003

[3] V. M. Abazov et al. (D0 Collaboration), Phys. Rev. D 84 (2011) 032004.

[4] ATLAS Collaboration, 2008 JINST 3 S08003.

[5] CMS Collaboration, 2008 JINST 3 S08004.

[6] CMS Collaboration, JHEP 12 (2012) 105.

[7] ATLAS Collaboration, ATLAS-CONF-2013-046, https://cds.cern.ch/record/1547327.

[8] ATLAS Collaboration, Eur.Phys.J. C 72 (2012) 2046.

[9] ATLAS Collaboration, ATLAS-CONF-2013-002, https://cds.cern.ch/record/1504739.

[10] ATLAS Collaboration, Eur.Phys.J. C 72 (2012) 2043.

[11] CMS Collaboration, CMS PAS TOP-12-029, https://cds.cern.ch/record/1521357.

[12] CMS Collaboration, CMS PAS TOP-12-031, https://cds.cern.ch/record/1528156.

[13] CMS Collaboration, CMS PAS TOP-12-035, https://cds.cern.ch/record/1520879.

[14] T. Aaltonen et al. (CDF Collaboration), Phys. Rev. D 87111101 (2013).

[15] CMS Collaboration, CMS PAS TOP-12-037, https://cds.cern.ch/record/1546763.

[16] ATLAS Collaboration, JHEP 1209 (2012) 139.

[17] CMS Collaboration, CMS PAS TOP-11-028, https://cds.cern.ch/record/1429971?ln=en.

[18] J. A. Aguilar-Saavedra and J. Bernabeu, Nucl. Phys. B 840 (2010) 349.

[19] ATLAS and CMS Collaborations, ATLASCONF-2013-033, CMS PAS TOP-12-025, https://cds.cern.ch/record/1527531.

[20] CMS Collaboration, CMS PAS TOP-11-020.

[21] ATLAS Collaboration, ATLAS-CONF-2011-037, https://cds.cern.ch/record/1337787.

[22] ATLAS Collaboration, JHEP 1206 (2012) 088.

[23] L. Lyons, D. Gibaut, and P. Clifford, Nucl. Instrum. Meth. A 270 (1988) 110.

[24] A. Valassi, Nucl. Instrum. Meth. A 500 (2003) 391.

[25] CMS Collaboration, CMS PAS TOP-12-015, https://cds.cern.ch/record/1523682.

[26] CMS Collaboration, CMS PAS TOP-12-020, https://cds.cern.ch/record/1523619. 
[27] T. Aaltonen et al. (CDF and D0 Collaborations), Phys. Rev. D 85, 071106 (2012).
[28] ATLAS Collaboration, ATLAS-CONF-2013-032, https://cds.cern.ch/record/1527128. 